\title{
Magnetic field causes the strong and weak nuclear forces and is the GUT force
}

\author{
John C. Hodge ${ }^{1 *}$ \\ ${ }^{1}$ Retired, 477 Mincey Rd., Franklin, NC, 28734
}

\begin{abstract}
The Scalar Theory of Everything (STOE) suggests the four fundamental forces are emergent properties of the magnetic field of permanent magnets. The STOE postulates the fractal (self-similar) principle that suggests small size scales are similar to our everyday size scale. Thus, the assembly of permanent magnets into a sphere is similar to a nucleon. The magnetic force from the sphere varies as the inverse cube of the distance from the center of the sphere (nucleon) which is approximately exponential, it has short range attractive field, it is an assembly of gluons (short photons), it explains the one proton to one electron atomic structure despite the large difference in mass, it suggests a size limit to the nucleon, it reminds of strong and weak forces, and it suggests a structure of the nucleus has protons on an outside shell. The STOE has the feature of the other sense of a "Theory of Everything" - that of a "Grand Unified Theory" (GUT).
\end{abstract}

keywords: magnet, GUT, strong force, weak force

\section{INTRODUCTION}

The Scalar Theory of Everything (STOE) posits the universe is composed of hods and plenum which emerge to form all in the universe (Hodge 2016b). The hods have the plenum highest density on one side and zero density on the other (Hodge 2018e, figure 3.1). That is, the hod is fundamentally a magnet with North and South poles. Magnetic monopoles are nonexistent. Electric effects are vortices formed in the plenum by the movement of hods through the plenum (Hodge 2018a).

The Scalar Theory of Everything (STOE) rejected the Biot-Savart Law and suggested two types of magnetic effects (Hodge 2018b,c,d). One type emanates from magnets, the other from electric currents.

The STOE suggests the self-similarity principle that posits small size scales are similar to our macro size scale. Accordingly, disk magnets were used to

*E-mail: jchodge@frontier.com 


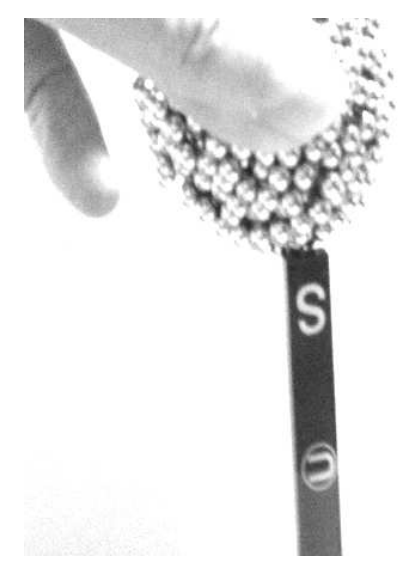

Figure 1: Sphere of ball magnets attracts the $\mathrm{S}$ pole of a bar magnet.

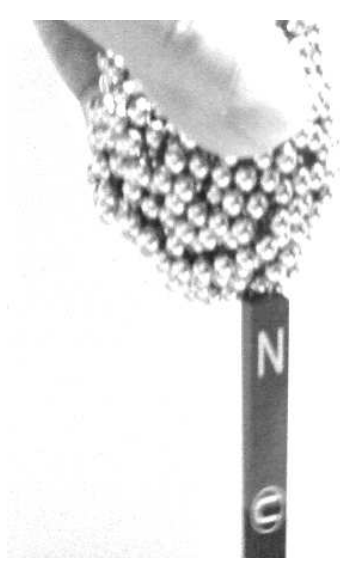

Figure 2: Sphere of ball magnets attracts the $\mathrm{N}$ pole of a bar magnet.

model photons and leptons (Hodge 2016a). This principle was used to suggest an atomic structure modeled as a structure of disk magnets (Hodge 2019b). According to the STOE, gravity is an emerged property (Hodge 2016b) of the plenum similar to the permanent magnetic effects of hods and not from an electric charge of Coulomb force (Hodge 2018a, 2019c).

Markoulakis (2020) noted that a sphere of small ball magnets could always attract other magnets as seen in Figs. 1 and 2.

This Paper describes a suggested proton structure of magnets using ball magnets to represent hods. The model of the nucleon is discussed in section 2 . The description of the experiment is in section 3. The possible structures are described in section 4. The Discussion and Conclusion are in section 5 .

\section{THE MODEL}

Figures 3 and 4 shows bar magnets aligned such that one approaches the center of the other. The lower magnet slips to the side such that there is net attraction. This is the mechanism of the sphere of ball magnets (Hodge 2019d).

However, this attraction differs from the long-distance (gravity) effect for molecules and larger (Hodge 2019c) and from the atomic structure effect (Hodge 2019b).

Examination of the balls in the sphere reveals that the $\mathrm{N}-\mathrm{S}$ axis of each ball magnet is approximately perpendicular to the radius of the sphere (Hodge $2019 \mathrm{~d}$ ). That is, the force of attraction along the radius of the sphere is approximately that perpendicular to a magnetic dipole. The force reduces with the inverse cube of the distance from the surface of the sphere. The ball magnets internal to the sphere are so aligned to reduce their effect external to the sphere. 


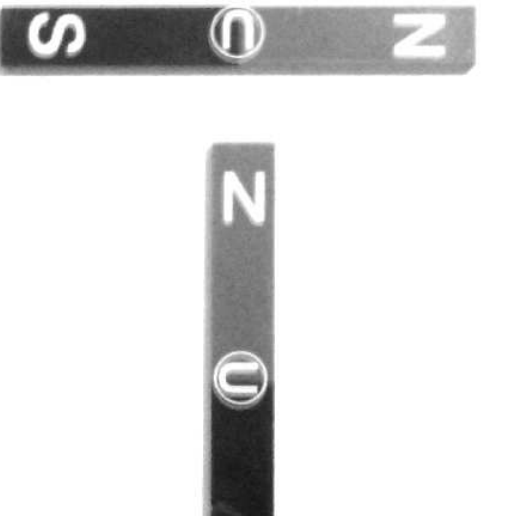

Figure 3: Bar magnets with one approaching the center of the other.

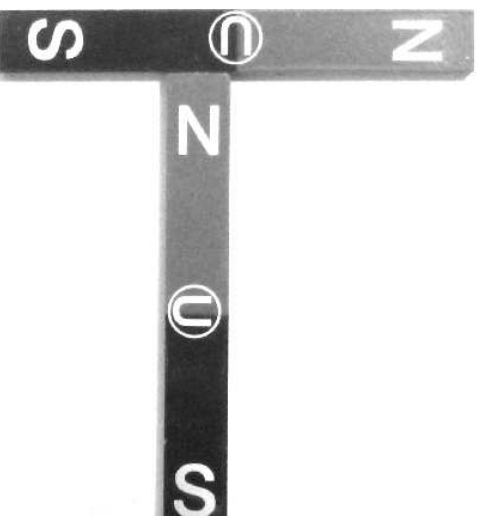

Figure 4: Bar magnets with one slipped off-center by the magnetic attraction.

Table 1: $W$ vs. $D$.

\begin{tabular}{|c|c|l|c|l|}
\hline \hline & \multicolumn{2}{|c|}{ inverse cube } & \multicolumn{2}{|c|}{ exponential } \\
\hline $\begin{array}{c}\text { No. } \\
\text { balls }\end{array}$ & $\begin{array}{c}\text { No. } \\
\text { pts. }\end{array}$ & $\begin{array}{l}W \text { Fig. } 6 \\
(\mathrm{gw})\end{array}$ & $\begin{array}{c}\text { No. } \\
\text { pts }\end{array}$ & $\begin{array}{l}W \text { Fig. } 7 \\
(\mathrm{gw})\end{array}$ \\
\hline 125 & 4 & $\left(95.6 * 1000 / D^{3}-9.8\right) \pm 0.2$ & 4 & $e^{-0.31 D+7.3} \pm 2$ \\
250 & 4 & $\left(98.1 * 1000 / D^{3}-2.2\right) \pm 0.05$ & 4 & $e^{-0.6 D+16} \pm 0.7$ \\
500 & 4 & $\left(734 * 1000 / D^{3}-16.5\right) \pm 2$ & 4 & $e^{-0.29 D+11} \pm 1.5$ \\
900 & 4 & $\left(748 * 1000 / D^{3}-16.4\right) \pm 0.1$ & 3 & $e^{-0.5 D+18} \pm 0.06$ \\
\hline
\end{tabular}

These magnets can form smaller particles such as seen in photons, neutrinos, and electrons (Hodge 2016a).

\section{THE EXPERIMENT}

The diagram in Figure 5 shows the experimental setup. Ball magnets $(5 \mathrm{~mm}$ diameter) are used to make spheres of ball magnets. The same experiment setup and procedure that was used in Hodge (2019a) is used in this experiment. The top wood support is lowered to change the distance between magnets $d$. The distance between the center of the sphere and the magnet $D=d+B / 2 \mathrm{~mm}$ (see Fig. 5).

The scale is "zeroed" by weighing the lower assembly without the top magnet in position. The weight measured $W$ gram weight (gw) is with the top magnet in position. Therefore, the $W$ is the force exerted by the top magnet on the bottom magnet.

(All measurement are $\pm 10 \%$ unless otherwise stated). 


\section{THE EXPERIMENT}

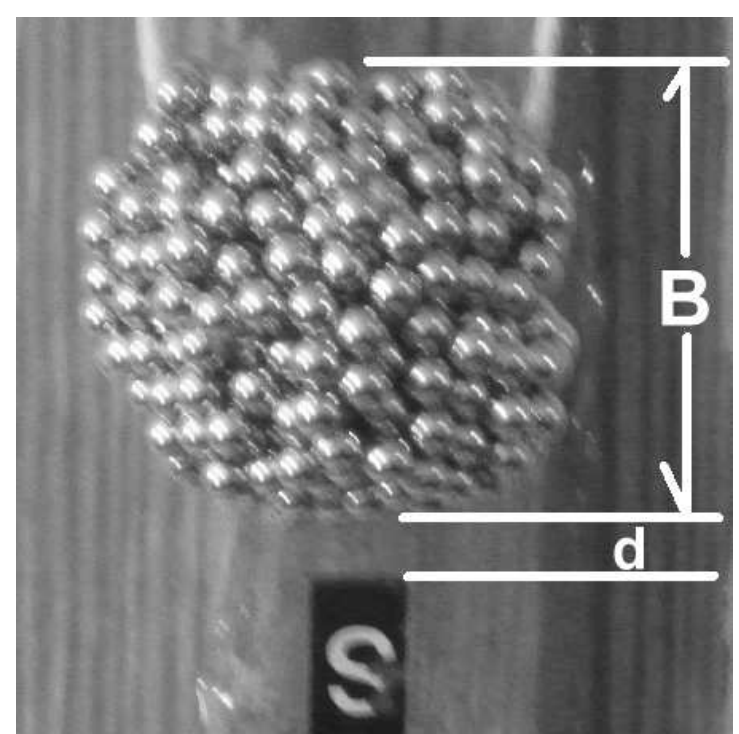

Figure 5: Photo of the experimental fixtures showing the sphere and bar magnet.

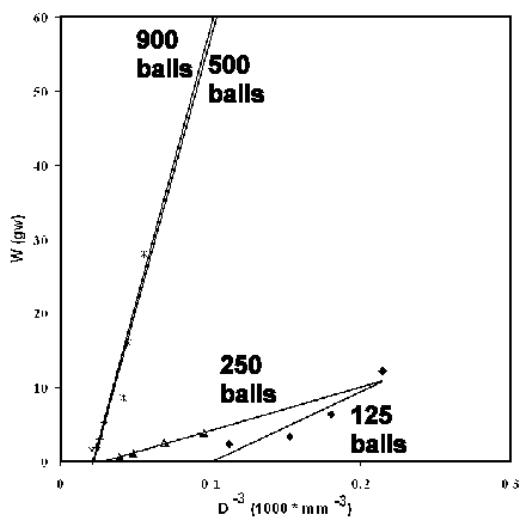

Figure 6: Graph of $W$ vs. $D^{-3}$ for various Sphere sizes showing the linear regression best fits.

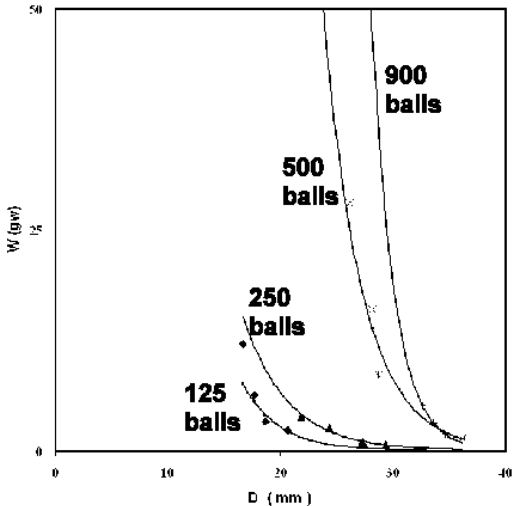

Figure 7: Graph of $W$ vs. $D$ for various Sphere sizes showing the exponential best fits. 


\section{POSSIBLE STRUCTURES}

The inverse cube of the force from the sphere is a slightly better fit to the data than the exponential. This was found in the initial paper on the magnetic field evolving to gravity (Hodge 2019a) and is a characteristic of magnets with the N-S pole axis perpendicular to the measurement. Gravity, which is a mismatch to the magnetic pole strength at distances larger than the atomic scale (Hodge 2019c), is considerably smaller than the magnetic strength that is still present in the nucleus.

Figure 8 shows a possible structure with the proton magnetically attached to a neutron (the slightly smaller sphere on the left). This causes a slightly high magnetic attaching force on the opposite side where a photon [a column of hods / magnets (Hodge 2016a)] is attached. Hodge (2012) and several experiments show this model of a photon are not rejected by several experiment that reject wave models of interference.

Figure 9 shows the result if another photon is attracted to the proton. The photons magnetically join to become one entity to connect to the electron on the other end of the photon (Hodge 2016a).

Figure 10 shows the result if the photon is too short or of insufficient energy. The other end is also attracted to the sphere/proton and the connection to the electron fails.

Figure 11 shows that other subatomic particles (the small sphere) or neutron become attached.

Figure 12 shows what happens if the sphere is pulled apart. Note the short, photon-looking structures with each end terminating on the sphere. These are suggested to be the analogy to gluons inside the protons and neutrons.

By suggesting a magnetic dominated structure, the model is including the tendency toward minimum potential energy as the guiding consideration in forming structures. If another proton neutron pair becomes attached, the proton must have a side facing outward to attach an electron through the photon. The only stable structure is for the neutron to attach to the neutron and the proton to the proton.

A longstanding problem in both nuclear structure and atomic structure is the electric concept of the one proton to one electron compared to the large mass difference. The charge model of the atom suggests the proton should have an equal but opposite charge of the electron. The large mass difference would seem to suggest a charge difference. The magnetic strength holds the nucleus and the atomic structure together (Hodge 2019b) with photons holding one electron to one proton. Further, the experiment shows the inverse cube force is very short range, less than the a tenth of the diameter of the sphere. For a proton this is about $0.1 \mathrm{fm}$. in agreement with findings for the strong force. 


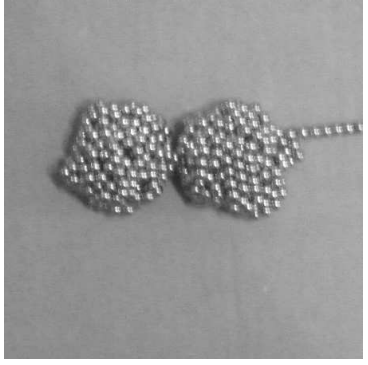

Figure 8: Possible structure of a proton, neutron, and photon attachment.
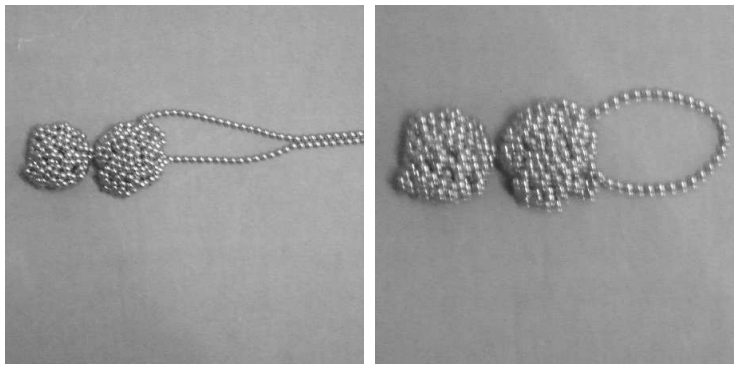

Figure 9: What hap- Figure 10: What happens when another pho- pens when the photon is ton tries to attach to too short, less than sufthe proton. ficient energy.

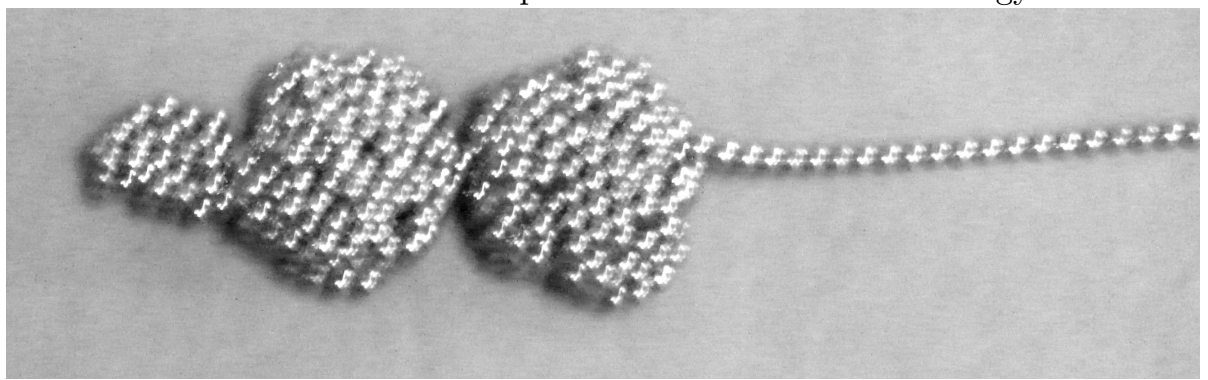

Figure 11: Other subatomic particles or neutrons can become attached to the neutron side.

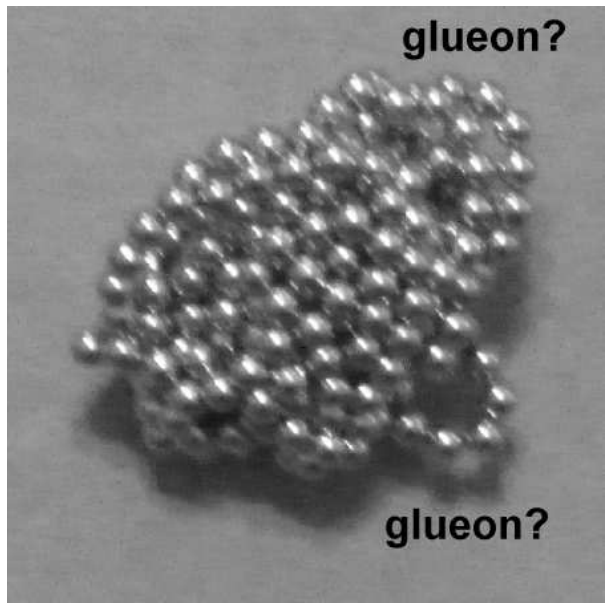

Figure 12: What happens when a sphere is pulled apart showing possible glueons. 


\section{DISCUSSION AND CONCLUSION}

The attractive effect of the proton is not the same as the long-distance (gravity) effect for molecules and larger (Hodge 2019c) and not the same as the atomic structure effect of Hodge (2019b). The magnetic ball sphere has the attractive force similar to the force expected by the Gauge Theory. That is, it is shorter range than the diameter of the sphere and is close to the exponential falloff with distance. Further, the interior structure of the sphere is of series of N-S magnetic coupling and appears as short photons. These bear the resemblance of gluons in the Gauge Theory.

More neutron-proton pairs combine to form a shell with protons on the outside and neutrons on the inside - a hollow sphere where other neutrons may collect. So, protons on the outside allow the electron to attach through photons one electron to one proton for stability, as observed. Note this is a considerable change to the model of a nucleus as being a jumble of nuclei.

This structure may offer a means to predict experiments. An extra neutron for large nuclei could cause an imbalance or a need to grow in size not allowed by the proton shell dimensions. So, fission results. Radioactive decay may also be a means to study the suggested structure. At least this model may suggest a method to evaluate radioactive decay whereas current model consider the topic an unresolvable problem. Such mechanisms are unknown but the results are well chronicled.

On the other hand, this speculation is consistent with the STOE model that gravity, the strong force, and the weak force are emergent effects of the plenum.

The suggested toy model of a nucleus has the potential to relate several and unexplained problem observations.

The STOE suggests the magnetic force is the Grand Unified Theory (GUT) that unites the four fundamental forces. The STOE began as a single model of both the big of cosmology and the small. The STOE postulates the fractal (self-similar) principle that suggests small size scales are similar to our everyday size scale. Thus, the assembly of permanent magnets into a sphere is similar to a nucleon. The present magnetic model of the nucleus exhibits many properties of the nucleus and atomic structure. Thus, it may serve as a model to discover other characteristics of nuclear structure. The magnetic force from the sphere varies as the inverse cube of the distance from the center of the sphere (nucleon), it has short range attractive field, it is an assembly of gluons (short photons), it explains the one proton to one electron atomic structure despite the large difference in mass, it suggests a size limit to the nucleon, it reminds of strong and weak forces, and it suggests a structure of the nucleus has protons on an outside shell. The STOE has the feature of the other sense of a "Theory of Everything" - that of a "Grand Unified Theory" (GUT). 


\section{REFERENCES}

\section{References}

Hodge, J.C., 2012, Photon diffraction and interference, IntellectualArchive, Vol.1, No. 3, P. 20, http://intellectualarchive.com/?link=item\&id=597

Hodge, J.C., 2016a, Structure and spin of the neutrino, electron, and positron, IntellectualArchive, Vol.5, No. 5, P. 1,. http://intellectualarchive.com/?link=item\&id=1694

Hodge, J.C., 2016b, STOE emergence, http://intellectualarchive.com/?link=item\&id=1757

Hodge, J.C., 2018a, STOE electric charge, IntellectualArchive, Vol.7, No. 2, P 1. 1 , http://intellectualarchive.com/?link=item\&id=1917

Hodge, J.C., 2018b, Magnetostatics relation to gravity with experiment that rejects Biot-Savart Law, IntellectualArchive, Vol.7, No. 3, P. 1,. http://intellectualarchive.com/?link=item\&id=1945

Hodge, J.C., 2018c, Another experiment rejects Ampere's Law and supports the STOE model, IntellectualArchive, Vol.7, No. 4, P. 6,. http://intellectualarchive.com/?link=item\&id=1956

Hodge, J.C., 2018d, Two different types of magnetic field, IntellectualArchive, Vol.4, No. 4, P. 1,. http://intellectualarchive.com/?link=item\&id=1964

Hodge, J.C., 2018e, STOE replaces relativity and quantum mechanics, (ISBN 978-613-9-91465-4, (LAP LAMBERT Acedemic publishing, Mauritius, available through Amazon.com).

Hodge, J.C., 2019a, Magnetic field evolves to gravity field Part 1: Repulsion , http://intellectualarchive.com/?link=item\&id $=2164$

Hodge, J.C., 2019b, Magnetic field evolves to gravity field part:4 Atomic structure, IntellectualArchive, Vol.8, No. 4, P. 30,. http://intellectualarchive.com/?link=item\&id=2199

Hodge, J.C., 2019c, Magnetic field evolves to gravity field part:5 Final, IntellectualArchive, Vol.8, No. 4, P. 37,. http://intellectualarchive.com/?link=item\&id=2233

Hodge, J.C., 2019d, video: https://www.youtube.com/watch?v=B1uGyD8uNwM

Markoulakis, E. 2020, personal communications in response to Hodge (2019b). https://www.youtube.com/watch?v=HZrv7V41qm0 https://www.youtube.com/watch?v=utlnD0yhmA8 